
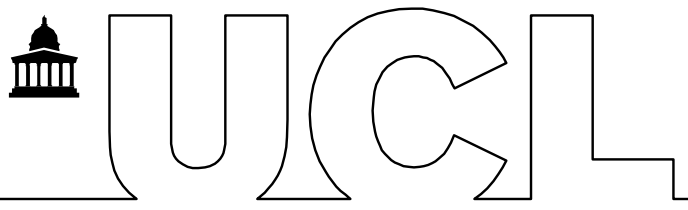

Shakespeare, TJ; Pertzov, Y; Yong, KXX; Nicholas, J; Crutch, SJ; (2015) Reduced modulation of scanpaths in response to task demands in posterior cortical atrophy. Neuropsychologia, 68 pp. 190200. 10.1016/i.neuropsychologia.2015.01.020. Downloaded from UCL Discovery: http://discovery.ucl.ac.uk/1461985

\title{
ARTICLE
}

\section{Reduced modulation of scanpaths in response to task demands in posterior cortical atrophy}

\author{
Timothy J. Shakespeare ${ }^{a^{*}}$, Yoni Pertzov ${ }^{b}$, Keir X.X. Yong ${ }^{a}$, Jennifer Nicholas ${ }^{c}$, Sebastian J. \\ Crutch $^{\mathrm{a}}$ \\ ${ }^{a}$ Dementia Research Centre, Department of Neurodegenerative Disease, Institute of \\ Neurology, University College London, London, UK \\ ${ }^{b}$ Institute of Cognitive Neuroscience, University College London, London, UK \\ ${ }^{c}$ Department of Medical Statistics, London School of Hygiene and Tropical Medicine, \\ London, UK \\ * Corresponding author: Dr Timothy Shakespeare, Dementia Research Centre, Box 16, \\ National Hospital for Neurology and Neurosurgery, Queen Square, London, WC1N 3BG, \\ UK; e-mail: tim.shakespeare@ucl.ac.uk; telephone: +44203448 3612
}

\begin{abstract}
A difficulty in perceiving visual scenes is one of the most striking impairments experienced by patients with the clinico-radiological syndrome posterior cortical atrophy (PCA). However whilst a number of studies have investigated perception of relatively simple experimental stimuli in these individuals, little is known about multiple object and complex scene perception and the role of eye movements in posterior cortical atrophy. We embrace the distinction between high-level (top-down) and low-level (bottom-up) influences upon scanning eye movements when looking at scenes. This distinction was inspired by Yarbus (1967), who demonstrated how the location of our fixations is affected by task instructions and not only the stimulus' low level properties. We therefore examined how scanning patterns are influenced by task instructions and low-level visual properties in 7 patients with posterior cortical atrophy, 8 patients with typical Alzheimer's disease, and 19 healthy agematched controls.
\end{abstract}

Each participant viewed 10 scenes under four task conditions (encoding, recognition, search and description) whilst eye movements were recorded. The results reveal significant differences between groups in the impact of test instructions upon scanpaths. Across tasks without a search component, posterior cortical atrophy patients were significantly less consistent than typical Alzheimer's disease patients and controls in where they were looking. By contrast, when comparing search and non-search tasks, it was controls who exhibited lowest between-task similarity ratings, suggesting they were better able than posterior cortical atrophy or typical Alzheimer's disease patients to respond appropriately to high-level needs by looking at task-relevant regions of a scene. Posterior cortical atrophy patients had a significant tendency to fixate upon more low-level salient parts of the scenes than controls irrespective of the viewing task. The study provides a detailed characterization of scene perception abilities in posterior cortical atrophy and offers insights into the mechanisms by which high-level cognitive schemes interact with low-level perception. 
Keywords: Alzheimer's disease; eye tracking; neurodegenerative disorders; visual agnosia.

\section{Highlights:}

- We investigated eye movements of PCA patients when viewing scenes.

- The effects of bottom-up and top-down influences were examined.

- PCA patients fixated upon regions of greater low-level salience than controls.

- PCA patients showed reduced task appropriate modulation of scanpaths.

\section{Introduction}

In 1967, the English translation of the Russian biophysicist Alfred Yarbus' landmark book Eye Movements and Vision brought to wide attention his innovative studies of human eye movement behaviour (Yarbus, 1967; see Tatler et al., 2010). In perhaps the most often-cited experiment, Yarbus presented a participant with Repin's An Unexpected Visitor under seven different task conditions (free examination, remember the clothes worn by the people, estimate the material circumstances of the family in the picture, give the ages of the people, describe what the family was doing before the visitor arrived, remember where the objects are in the room, estimate how long the visitor had been away). From this qualitative but nonetheless compelling data, Yarbus concluded '...that the distribution of the points of fixation on an object, the order in which the observer's attention moves from one point of fixation to another, the duration of the fixations, the distinctive cyclic pattern of examination, and so on are determined by the nature of the object and the problem facing the observer at the moment of perception' (Yarbus, 1967, p196). Together with Buswell's (1935) demonstration of the effect of test instructions upon viewing behaviour, Yarbus's work stimulated a continuing controversy over the mechanisms by which low-level image features and higher-level cognitive representations shape the way we view and understand the world around us. In the current study, we apply Yarbus' paradigm to individuals with primary neurodegeneration of the visual cortices (posterior cortical atrophy; PCA; Benson et al., 1988; see Crutch et al., 2012 for a review) to provide a novel perspective on the debate he inspired and to use his methodology to better understand how these profoundly visuallyimpaired individuals perceive their environment.

Scene perception involves the acquisition of high quality information from the region surrounding the centre of gaze during brief periods of relative stability (fixation) before gaze is re-oriented to another area of the scene by means of a rapid eye movement (saccade). Fixations are not randomly placed within a scene, but rather are clustered around areas of a scene which are informative to the viewer's current perceptual, cognitive or behavioural activity (Henderson, 2011). Two broad classes of explanation have been provided as to how the location of fixations within a scene are determined: low-level image features and highlevel knowledge structures (Henderson, 2007). Low-level image features include scene components such as colour contrast and orientation of edges (Koch and Ullman 1985; Itti and Koch, 2000, 2001). These low-level features, and more specifically their spatial contrast from the surrounding, can be combined to compute maps that model the low-level salience across an image (known as saliency maps). High-level knowledge structures (or prior knowledge) include short-term episodic scene knowledge (e.g. my cup is currently on my desk), long-term episodic scene knowledge (e.g. my coffeemaker always sits on shelf under the window), scene scheme knowledge (e.g. office computers are typically found on desks) and task knowledge (e.g. changing lanes while driving requires checking the side-view mirror; see Henderson and Ferreira, 2004; Henderson, 2011). Another factor known to affect the location of fixations when viewing pictures is the central fixation bias (Parkhurst et al., 2002; Tatler et al., 2005), by which observers tend to fixate regions of the picture towards the centre of the screen, and this has been demonstrated to occur irrespective of the task or the distribution of image features within a scene (Tatler, 2007).

Whilst there is debate about the timing and relative contribution of low- and high-level influences on gaze direction especially during the first few fixations following scene 
presentation (e.g. Mannan et al., 2009; Foulsham et al., 2011), most authors acknowledge that image features and knowledge structures have to be combined. The interaction of these factors in determining viewing behaviours has been the subject of a small number of studies (see Chen et al., 2006; Navalpakkam et al., 2005; Foulsham et al., 2009 \& 2011; Rao et al., 2002; Torralba et al., 2006; Henderson et al., 2009). In the current experiment we attempted to examine the interaction between high- and low-level influences in two ways. First, by administering a Yarbus-style paradigm (varying task whilst holding image features [scene] constant) to individuals with and without basic perceptual impairment and testing whether the effect of the task differs between the participant groups. Second, more directly, by examining how participants' scanpaths during different tasks corresponded to saliency maps of the scenes being viewed and testing for differences in the saliency effect across both task and group. The primary comparison of interest was between search and non-search tasks.

A limited number of studies have examined high-level influences upon scene perception using the Yarbus paradigm. This work has either been concerned with replicating the original single-case observations in a quantitative group study (DeAngelus and Pelz, 2009) or identifying which eye movement parameters are affected by task (in normal participants, task has been found to influence selection of scene regions but not duration of individual fixations; Castelhano et al., 2009; and differences between encoding and recognition have been described; Foulsham et al., 2012). Comparable studies of task influence upon scene perception in neurological populations are limited (free viewing versus search in hemispatial neglect patients; Machner et al., 2012) although some case studies of visual agnosia have alluded to the importance of task effects (e.g. Foulsham et al. 2009 \& 2011).

Here we examine influences upon the gaze behaviour of a group of patients with PCA. PCA is a clinicoradiological syndrome characterized by a gradual loss of visual skills and other posterior cortical functions due to atrophy of the parietal, occipital and occipitotemporal regions of the brain (Mendez et al., 2002; Tang-Wai et al., 2004; Lehmann et al., 2011). Patients with PCA demonstrate relatively spared episodic memory function in conjunction with prominent impairments of space perception, object perception, alexia, agraphia, acalculia, apraxia and some or all of the features of Balint's syndrome (simultanagnosia, oculomotor apraxia, optic ataxia, environmental agnosia; Mendez et al., 2002; Renner et al., 2004; Tang-Wai et al., 2004; Charles and Hillis, 2005; McMonagle et al., 2006, Lehmann et al., 2011). The majority of cases of PCA are caused by Alzheimer's disease (AD) (Renner et al., 2004; Tang-Wai et al., 2004). PCA is typically a young onset condition, most commonly emerging in ages 50 s and early $60 \mathrm{~s}$, but the exact prevalence and incidence remain to be established.

The complementary goals of the study were to characterise scene perception deficits in PCA and typical $A D$, and in doing so shed light on the mechanisms by which high-level cognitive schemes interact with low-level perception. A critical distinction between this study and previous studies employing the Yarbus paradigm was our aim not only to demonstrate task effects upon eye movement behaviour but to examine how the extent and nature of those task effects differed between healthy individuals and two neurodegenerative disease populations (PCA and typical $A D$ ). The null hypothesis was that the visual dysfunction observed in PCA would have a uniform effect upon scene perception across search and non-search tasks. The alternative hypothesis was that despite holding perceptual demands constant (same scenes), individuals with varying levels of basic visual dysfunction (grave PCA; mild - typical AD; no deficits - controls) would respond differently to search and nonsearch tasks. The interaction between low-level image features and high-level knowledgedriven control of scene perception was then tested directly by comparing participants' scanpaths under different task conditions to low-level salience maps generated for each photograph. 


\section{Methods}

\subsection{Participants}

Data were collected from seven PCA patients, a disease control group consisting of eight patients with typical AD (tAD) and 19 healthy controls, recruited from research cohorts at the Dementia Research Centre, UCL. PCA patients fulfilled standard clinical criteria for PCA (Mendez et al., 2002; Tang-Wai et al., 2004), had a clinical diagnosis of Alzheimer's disease (Dubois et al., 2007, 2010) and scored in the normal range $\left(>5^{\text {th }} \%\right.$ ile) on the short Recognition Memory Test for words (sRMT; Warrington, 1996) at the time of assessment. tAD patients (4 male) fulfilled Dubois criteria for $A D$ and scored in the impaired range $\left(<5^{\text {th }}\right.$ \%ile) on the RMT for words. An additional eligibility criterion of mild-moderate disease severity (MMSE score greater than 15/30) was applied to both patient groups. This project was approved by the NRES Committee London - Queen Square, according to guidelines established by the Declaration of Helsinki.

\subsection{Stimuli and procedure}

1.2.1. Background saccade-gain task

Participants completed a background pro-saccade (visually guided saccade) task to identify basic differences in oculomotor function and provide a saccade-gain covariate for the main scene perception task. A fixation dot subtending $0.4^{\circ}$ was presented at the centre of the screen for $500 \mathrm{~ms}$, followed by a target stimulus subtending $0.8^{\circ}$ for $5000 \mathrm{~ms}$ or until the target had been fixated for $250 \mathrm{~ms}$. Targets were presented at $5^{\circ}$ of eccentricity, left, right, up, or down from the fixation dot (8 trials in each direction). In half the trials target onset occurred $200 \mathrm{~ms}$ after fixation point offset (gap condition), and in half target onset occurred $200 \mathrm{~ms}$ prior to fixation offset (overlap condition). These trials were collected as part of a wider experiment that consisted of a total of 80 trials split over 4 blocks including targets presented at $10^{\circ}$ and $15^{\circ}$ of eccentricity. Here, we use the results of saccades to targets presented at $5^{\circ}$ as these more closely match the amplitudes of saccades made by control participants during scene perception (see results of saccade amplitude in the scene task). Each trial was preceded by a centrally-presented fixation point used as a drift correct stimulus, with the trial initiated by the experimenter.

\subsubsection{Scene stimuli}

The stimuli were 30 photographic images of street scenes selected from a readily available dataset (Ehinger et al., 2009; see supplementary material S1). Stimulus resolution was $800 \times 600$ pixels and images subtended a visual angle of $19.5^{\circ} \times 14.9^{\circ}$ from a fixed viewing distance of $60 \mathrm{~cm}$. All photographs were taken from a conventional human perspective with all elements conforming to standard physical (e.g. gravity, space) and semantic (e.g. cars located on roads not on buildings or trees) constraints. Vegetation was selected as the search target (see below) in order to employ perceptually variable targets which could appear in multiple locations anywhere in a scene rather than a homogenous stimulus category whose potential locations were more circumscribed (e.g. faces).

\subsubsection{Scene Procedure}

Scene stimuli were presented under four different task conditions (encoding, recognition, search and verbal description). For the purposes of analysis, these four tasks were split into two conditions - non-search (encoding, recognition, verbal description) and search. We analysed the data based on this search/non-search dichotomy as we were interested to investigate differences between search, where the instruction required participants to fixate upon previously defined regions based on the visual properties of the scene, and non-search where there was no such explicit instruction to fixate upon certain regions of the scene. More details on each of the tasks are given below.

1. Encoding: 10 images (scenes 1-10) were presented sequentially. The participant was instructed to remember the photographs. 
2. Recognition: The 10 images presented in the encoding task (scenes 1-10) were represented, interleaved with 10 novel images which acted as distractors (scenes 11-20). Participants were asked 'Did I show you this picture before?' and their responses were recorded. Thus the task comprised 'familiar' $(\mathrm{N}=10)$ and 'novel' $(\mathrm{N}=10)$ subconditions.

3. Search: Images presented at encoding were presented again together with 10 further novel images (scenes 21-30). The participant was instructed to "look at all the trees, grass and plants; all the vegetation in the scene". As with the recognition task, the task comprised 'familiar' $(\mathrm{N}=10)$ and 'novel' $(\mathrm{N}=10)$ subconditions.

4. Verbal description. Scenes 1-10 were presented and participants were asked to describe the scene. Owing to the homogeneity of the scenes (all street scenes) most participants offered a description of some or all of the features. Word finding difficulties were evident in most of the patient sample (see background psychology results supplementary material S2). For these reasons the content of verbal descriptions was not analysed.

All photographs were presented for 5 seconds each except in the description condition where photographs were presented for 20 seconds. As with the saccade task, each trial was preceded by a centrally-presented fixation point used as a drift correct stimulus, with the trial initiated by the experimenter. Images were presented in a fixed random order within each task, and tasks were administered to all participants in the same order. Example scanpaths under each of the four different tasks are shown in Figure 1.

Figure 1. Example scan paths of one healthy participant viewing scenes under different task conditions: encoding (remember the scene), recognition (novel/familiar judgment), search (look at the vegetation), and verbal description (describe the scene). Circles show the location of fixations, and numbers in circles show the serial order of the fixations over time. The highlighted area shows the target-area for the search task.

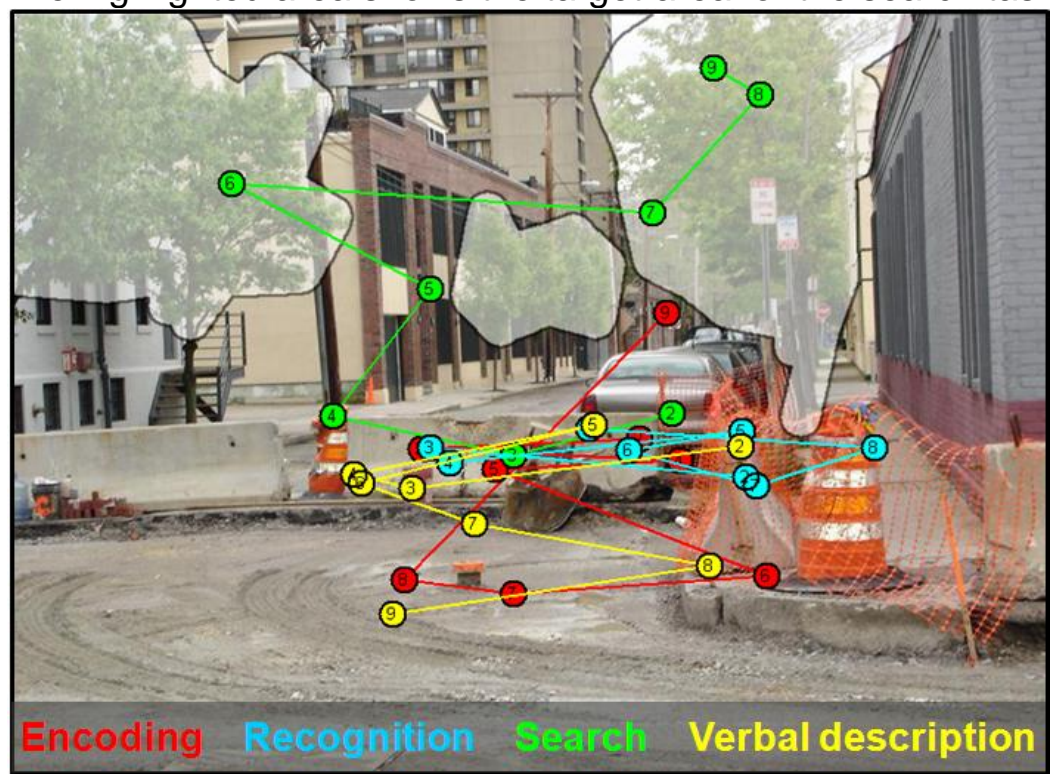

\subsubsection{Apparatus}

The saccade-gain and scene tasks were presented on a Dell 2120 desktop computer from a fixed viewing distance of $60 \mathrm{~cm}$. Eye movements were recorded using a head-mounted infrared video-based eye tracker (Eyelink II; SR Research, Canada). Gaze position was recorded at $250 \mathrm{~Hz}$ and corneal reflection was used when possible (17 participants). Fixations and saccades were parsed by the Eyelink system, using standard velocity and acceleration thresholds $\left(30 \% \mathrm{~s}\right.$ and $\left.8000 \% \mathrm{~s}^{2}\right)$. We used built-in programs provided with the eye tracker for calibration and validation purposes (five points presented in a random 
sequence). All the data analyzed were obtained from recordings with an average Cartesian prediction error of $<1^{\circ}$ during the validation procedures. Participants used a chin rest (wide HeadSpot; University of Houston College of Optometry) to provide stability and maintain viewing distance throughout the experiment.

\subsubsection{Pre-processing and analysis}

1.2.5.1. Background saccade-gain task

Analysis was carried out on the dominant saccade made towards the target. Data were cleaned to identify this saccade by removing: (i) blinks (using Eyelink's automated blink detection); (ii) saccades made before the target appeared; (iii) saccades in the wrong direction (saccade-target angle discrepancy $>45^{\circ}$ ); (iv) saccades starting $>5^{\circ}$ from the fixation point. Following this, only the first remaining saccade of each trial was kept. If this saccade was later than the $5^{\text {th }}$ saccade, the trial was removed. As a result, $6.9 \%$ of trials were removed in healthy control participants, compared to $6.3 \%$ of trials in tAD patients and $9.8 \%$ of trials in PCA patients. Linear regression was carried out in STATA to compare amplitude of the dominant saccade between groups, controlling for age. The mean gain of the dominant saccade (saccade amplitude / target amplitude) for each participant was saved for use as a covariate in later analyses.

We also investigated whether the relationship between peak saccade velocity and saccade amplitude differed between patient groups, by comparing peak saccadic velocity between groups, after controlling for saccade amplitude (amplitude was included a covariate in the linear regression; as in chapter 3).

\subsubsection{Scene task pre-processing}

Before data from the scene task were analysed, saccades identified by the Eyelink system as containing blinks were removed. All analyses excluded the first fixation of each trial (location determined by the pre-trial fixation point). The majority of analyses also excluded the 10th fixation onwards (as per Mannan et al., 2009) leaving fixations 2-9. The exceptions were basic saccade amplitude, fixation duration and the proportion of fixations within the target-area, where the data from the first five seconds of each trial were included.

\subsubsection{Scene task analysis}

Linear and logistic regression models (using repeated measures) were employed, with age and saccade-gain from the background saccade-gain task included as covariates of no interest in order to control for their potential influences (except for the analysis of fixation duration and recognition performance which were not corrected for saccade-gain). Due to skew in the distribution of fixation duration and saccade amplitude, analysis was carried out on the log transformation of the data, and atypical observations for each participant were discarded by excluding observations outside of two standard deviations of each participant group's mean.

The dependent variable in the analysis of performance on the recognition task was the number of items correct (/20). Dependent variables in the analysis of performance on the search task were proportion of fixations in target area and time to first fixation within the target-area. The experimenter (TS) created the target areas by manually drawing around the vegetation in each photograph to generate an in/out binary variable (see Figure 1); this conformed to the experimenter's best judgement of the boundary between vegetation and background but was not defined in terms of basic colour or intensity thresholds. Central fixation bias was examined by comparing the distance of fixations from the centre of the stimulus between groups and conditions. Two further analyses (index of similarity and scanpath saliency) merit more detailed consideration: 
1.2.5.4. Index of similarity

This analysis used the revised index of similarity metric described by Mannan et al., (2009; see supplementary material of that paper). This compares the spatial locations of fixations in any two scanpaths, but does not consider the order of fixations.

For each pair of scanpaths, each fixation from one scanpath is paired with a fixation from the other scanpath. Each combination of pairings is considered, and the combination that results in the minimum average squared difference in the location of the fixations between the pairs is chosen. The average squared difference between the paths for that combination is considered in a ratio with the average squared difference between scanpaths of the same observer to two different images (again using the combination of fixations providing the lowest average squared difference). This gives the index of similarity. Thus an index of similarity of $0 \%$ represents two scanpaths that are only as similar as eye movements made to two different images, whilst an index of similarity of $100 \%$ indicates that the two scanpaths had identical fixation locations. A detailed description of the method can be found in the supplementary material of Mannan et al., 2009. The index of similarity metric was used to measure the similarity of each participant's scanpath between repeated presentations of identical photographs under the four tasks (between-task similarity), and the similarity of each participant's scanpaths to those of every other participant for the same task and picture (between-observer similarity). The between-task similarity metric was calculated for each possible task-pairing of scenes 1-10 (i.e. encoding-recognition, encoding-feature search, recognition-feature search, recognition-description, and feature search-description). Between-observer similarity was calculated both within group (each participant to each other participant in the same group; i.e. PCA vs PCA, TAD vs tAD and control vs control) and between group (each participant from one group to each participant in another group; i.e. PCA vs control, tAD vs control, PCA vs tAD).

\subsubsection{Normalised scanpath saliency}

Each photograph was analysed using the graph-based visual salience (GBVS) toolbox (Harel et al., 2007) to generate a low-level salience map (see Figure 4). This toolbox was chosen due to its improved prediction over classical algorithms (e.g. Itti et al., 1998). The tool creates three feature maps for each image (representing variation in colour, intensity and orientation), and then combines these feature maps into a master map representing the computed salience at each pixel. These maps were then normalized to have a mean value of 0 and standard deviation of 1 across pixels. The salience of each fixation was extracted and compared between groups and conditions.

\subsubsection{Statistical analysis}

The following statistical tests were carried out for all analyses except for scene recognition performance and proportion of fixations in the target area (analyses for these two outcomes are described in the next paragraph). Linear regression models with robust standard errors to adjust for repeated measures were used, with age and saccade-gain from the background saccade-gain task included as covariates of no interest (in order to control for their potential influences). The analysis of fixation duration did not include saccade-gain as a covariate. Wald tests were carried out to elucidate the main effects, pairwise comparisons of different levels of factors, and interactions.

Analysis of scene recognition performance and proportion of fixations in the target area used a logistic regression model with robust standard errors to adjust for repeated measures. Age was included as a covariate. Saccade-gain was included as an additional covariate for the analysis of proportion of fixations in the target area.

In the analysis of fixation duration and saccade amplitude, analysis was carried out on the log transformation of the data, and atypical observations for each participant were discarded 
by excluding observations outside 2 standard deviations of each participant group mean. All statistical analyses were conducted using STATA 12.

\section{Results}

\subsection{Patient characteristics}

PCA patients (two male; Mean [SD] age $=58.9$ [6.3] years) were significantly younger than tAD patients (four male; Mean [SD] age $=69.7$ [4.7] years; two sample t-test $p=0.002$ ), reflecting the fact that PCA is typically an early-onset condition. Healthy control participants (5 male; Mean [SD] age $=63.1$ [5.2] years) did not differ significantly in age from the PCA patients (two sample t-test $p=0.097$ ) but were younger than the tAD group $(p=0.005)$.

Patient groups were matched for disease duration (mean [SD] PCA = 3.31 [2.0] years; tAD = 4.34 [2.2] years, $p=0.34$ ) and MMSE (mean [SD] $P C A=22.6$ [2.57]; $t A D=22.6$ [4.50], $\mathrm{p}=0.98$ ). Performance of PCA patients on further neuropsychological tests is presented in the supplementary material (S2).

Biomarkers of molecular pathology (amyloid PET scan or CSF sample) were available in 3/7 PCA patients. These were supportive of underlying AD in two cases (see Table 1). The remaining case had a CSF profile that was not supportive of AD. CSF was available in 4/8 $t A D$ patients, with a profile supportive of $A D$ in three cases and borderline compatible with $A D$ in the remaining case.

Table 1. Biomarkers of molecular pathology in patients. For the purposes of biomarker interpretation, samples with a $A \beta_{1-42}<550 \mathrm{pg} / \mathrm{ml}$ and $T a u: A \beta$ ratio $>1$ were considered supportive of underlying AD pathology (+). One PCA participant had a CSF profile not supportive of $A D(-)$. One tAD patient had a CSF profile which was borderline compatible with underlying $A D(+/-)$.

\begin{tabular}{|llllllll|}
\hline Diagnosis & PCA & PCA & PCA & tAD & tAD & tAD & tAD \\
CSF total tau $(\mathrm{pg} / \mathrm{ml})$ & 787 & 310 & 841 & 289 & 843 & 828 & 800 \\
CSF A $\beta$ 1-42 $(\mathrm{pg} / \mathrm{ml})$ & 297 & 488 & 264 & 280 & 129 & 125 & 297 \\
CSF Tau:A $\beta$ ratio & 2.65 & 0.64 & 3.19 & 1.03 & 6.53 & 6.62 & 2.69 \\
Biomarker interpretation & + & - & + & $+/-$ & + & + & + \\
\hline
\end{tabular}

\subsection{Background saccade-gain task}

Mean saccade gain (amplitude of dominant saccade divided by distance of target from central fixation point) was lower in PCA patients (mean [SD] gain=0.75 [0.26]) than controls $(0.90$ [0.26]; $\beta=0.16, t(24)=2.89, p=0.007)$ or tAD patients $(0.97[0.24] ; \beta=0.25, t(13)=$ 3.72, $p=0.002)$. tAD patients showed a trend towards greater gain than controls $(\beta=0.09$, $t(25)=1.89, \mathrm{p}=0.07$ ). The abnormalities in saccade gain (reduced in PCA, moderately increased in tAD) justify the inclusion of saccade gain as a covariate in the subsequent analyses.

There was no difference saccade peak velocity (having accounted for saccade amplitude) between the healthy controls and PCA patients $(\beta=8.97, t(24)=0.53, p=0.60)$, or between the PCA and tAD patients $(\beta=23.95, t(13)=1.22, p=0.23)$, although the typical AD patients showed increased peak velocity compared to the healthy controls $(\beta=32.92, t(25)=2.70$, $\mathrm{p}=0.01$ ). 


\subsection{Scene task}

\subsubsection{Fixation duration}

PCA patients (mean $=272.0 \mathrm{~ms}, S D=35.9 \mathrm{~ms}$ ) did not differ from controls (mean $=261.6 \mathrm{~ms}$, $S D=24.1 \mathrm{~ms}$ ) $\beta=0.02, t(24)=0.35, p=0.73$ ) or $\mathrm{tAD}$ patients (mean $=287.6 \mathrm{~ms}, \mathrm{SD}=31.9 \mathrm{~ms} ; \beta$ $=0.09, t(13)=1.25, \mathrm{p}=0.22)$ in terms of fixation duration. However there was a trend towards longer fixations in the tAD group than control group $(\beta=0.11, t(25)=2.00$, $p=0.054)$. Further analysis showed that $t A D$ patients had significantly longer fixations than controls for the non-search tasks (encoding, recognition, description; $p=0.03$ ) but not for the search task $(p=0.16)$.

\subsubsection{Saccade amplitude}

Controlling only for age, the amplitude of saccades made by PCA patients when viewing scenes $\left(\right.$ mean $=2.73^{\circ}, \mathrm{SD}=1.83^{\circ}$ ) was less than controls (mean $=3.48^{\circ}, \mathrm{SD}=2.44^{\circ} ; \beta=$ $0.21, t(24)=4.16, \mathrm{p}<0.001$ ) and $\mathrm{tAD}$ patients (mean $=3.60^{\circ}, \mathrm{SD}=2.32^{\circ} ; \beta=0.28, t(13)=$ 4.37, $p<0.001)$. tAD patients did not differ significantly from controls $(\beta=0.07, t(25)=1.25$, $\mathrm{p}=0.22)$. When controlling for age and saccade gain, PCA patients' saccades remained significantly shorter than those of controls $(\beta=0.10, t(24)=2.15, p=0.04)$, but did not differ significantly from tAD $(\beta=0.11, t(13)=1.52, p=0.14)$; tAD patients and controls remain not significantly different $(\beta=0.003, t(25)=0.05, p=0.96)$.

\subsubsection{Central fixation bias}

Controlling only for age, PCA patients' fixations remained significantly closer to the centre of the scene images compared to tAD patients $(\beta=0.64, t(13)=2.53, p=0.02)$ and there was a a similar effect relative to controls $(\beta=0.527, t(24)=3.03, p=0.005)$. There was no difference in central fixation bias between tAD patients and controls $(\beta=0.11, t(25)=0.52$, $\mathrm{p}=0.61$ ).

However, when controlling for saccade gain in the background task, these group differences between PCA and tAD $(\beta=.17, t(13)=0.48, p=0.63)$ and PCA and controls $(\beta=0.25, t(24)$ $=1.34, p=0.19$ ) were no longer significant (see Figure 2). This indicates that it may be important to obtain an independent measure of saccade gain as a covariate that can be controlled for in future tests. 
Figure 2. Central fixation bias measured as the mean distance of fixations from the centre of the screen, both controlling (green) and not controlling (red) for saccade-gain. Bars show $95 \%$ confidence intervals.

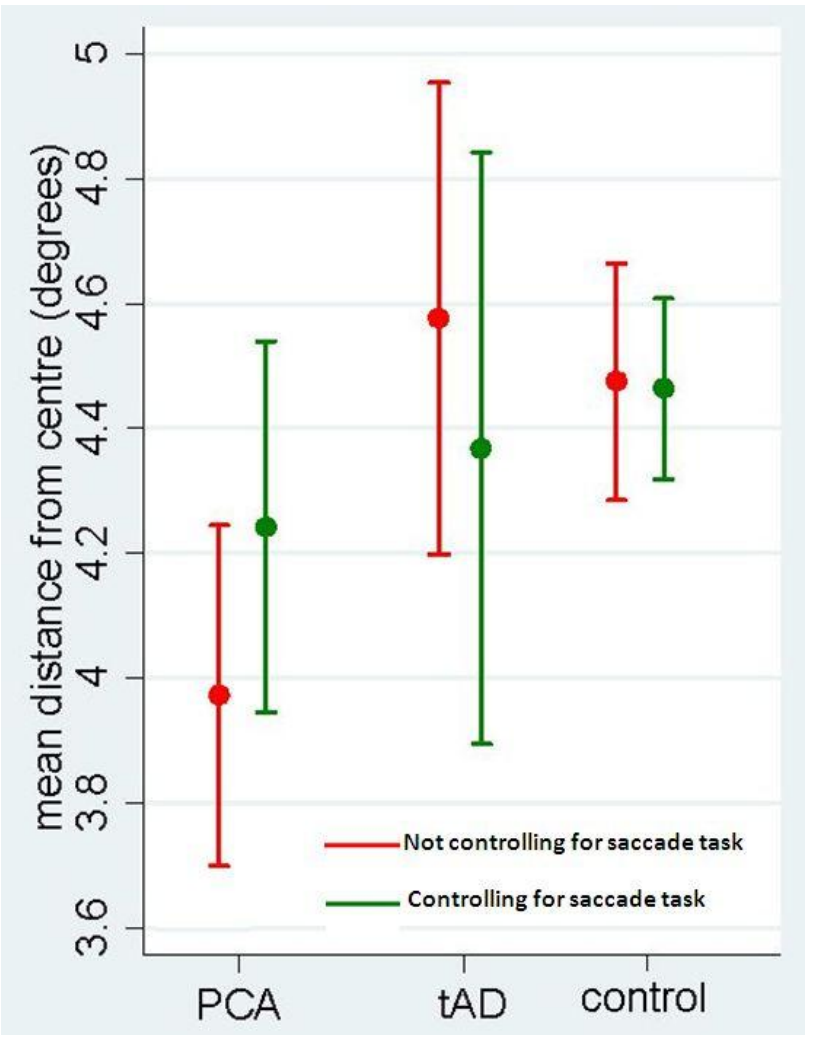

\subsubsection{Recognition task}

PCA (mean proportion correct $=0.81, S D=0.12$ ) and $\mathrm{tAD}$ patients (mean=0.81, $\mathrm{SD}=0.14$ ) showed poorer performance than controls (mean=0.98, $S D=0.04$ ) in the familiar/novel discrimination of the recognition task (PCA vs control $O R=0.11, z=4.43, p<0.001$; tAD vs control $\mathrm{OR}=0.11, z=4.34, \mathrm{p}<0.001)$. The performance of PCA patients and $\mathrm{TAD}$ patients did not differ significantly $(O R=0.99, z=0.03, p=0.98$; see Discussion for different explanations of scene recognition in these two groups).

\subsubsection{Search task}

PCA patients performed poorly in the search task, with a lower proportion of fixations in the target area (mean $=0.50, \mathrm{SD}=0.08$ ) than the control group (mean=0.66, $\mathrm{SD}=0.11 ; \mathrm{OR}=2.42$, $z=4.08, \mathrm{p}<0.001$ ). $\mathrm{tAD}$ patients (mean $=0.55, \mathrm{SD}=0.10$ ) also showed a non-significant trend towards a lower proportion of fixations within the target area than controls $(\mathrm{OR}=0.62, z=$ $1.87, \mathrm{p}=0.07)$. The PCA and tAD groups did not differ from one another significantly $(\mathrm{OR}=$ $1.51, z=1.21, p=0.22)$. There was a significant effect of novelty $(O R=1.92, z=7.54$, $p<0.001$ ), with a greater proportion of fixations within the target area for novel scenes. However, there was also a significant interaction between the effects of group and novelty $(\mathrm{p}=0.004$; see Figure 3); PCA patients showed a greater relative impairment when searching familiar as compared with novel scenes for the target 'vegetation', suggesting reduced benefit from previous exposure to and memory for the (familiar) scenes. There were also significant differences between groups in the time taken to make the first fixation in the target-area, with PCA patients much slower (mean $=845 \mathrm{~ms}, \mathrm{SD}=182 \mathrm{~ms}$ ) than controls (mean $=586 \mathrm{~ms}, S D=144 \mathrm{~ms} ; \quad \beta=257.21, t(24)=2.30, \quad \mathrm{p}=0.03$ ), and $\mathrm{tAD}$ patients (mean $=680 \mathrm{~ms}, S D=154 \mathrm{~ms}$ ) showing a trend towards slower performance than controls $(\beta=$ 135.96, $t(25)=1.81, p=0.08)$. $P C A$ and $t A D$ groups were not significantly different $(\beta=$ $121.25, t(13)=0.98, p=0.34)$. The main effect of novelty was significant, meaning the time taken to first fixation within the target area was shorter for novel scenes $(\beta=212.89, \mathrm{t}(32)=$ 
2.18, $\mathrm{p}=0.04)$. Unlike the search accuracy data, there was no evidence for an interaction between group and novelty in the search timing data $(p=0.75)$.

Figure 3. Performance in the search task in terms of proportion of fixations within the targetarea $(A)$ and time taken to make the first fixation within the target-area (B). Bars show 95\% confidence intervals.

A

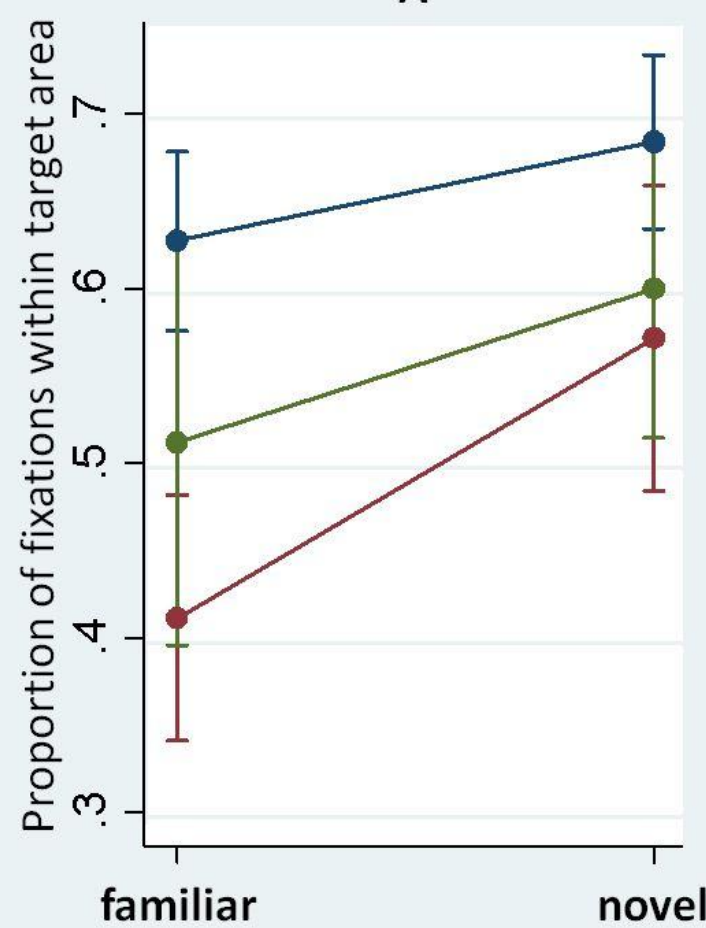

B

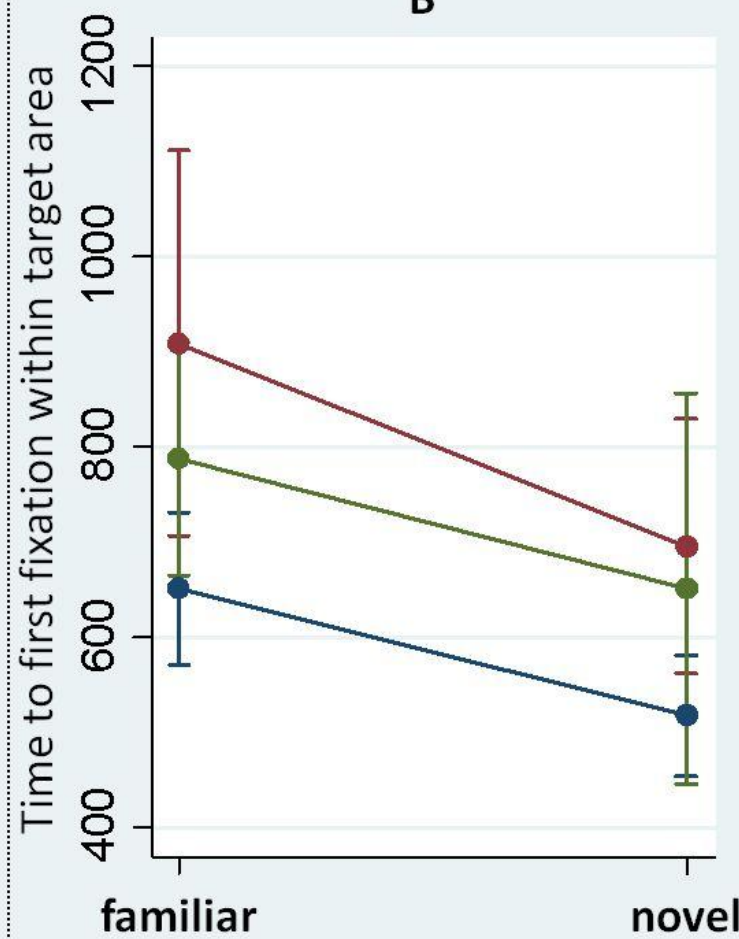

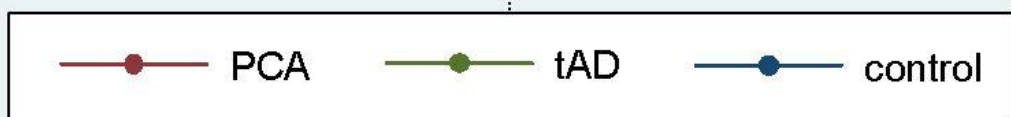

2.3.6. Between-task similarity

Between-task similarity measures the similarity of two scanpaths made by the same participant to identical scenes presented under different tasks (see Figure 1 and Figure 4 for example scanpaths, and Figure 5A for means and Cls). In the comparisons of non-search tasks (e.g. recognition vs description), PCA patients showed significantly lower scanpath consistency than controls $(\beta=15.85, t(24)=2.79, p=0.009)$ or tAD patients $(\beta=18.87, t(13)$ $=2.23, p=0.03$ ). Control participants and tAD patients did not differ in scanpath -consistency between non-search tasks $(\beta=3.02, t(25)=0.49, p=0.63)$. However, when comparing search and non-search tasks (e.g. search vs recognition); controls demonstrated a taskappropriate difference in scanpaths (reflected in zero consistency - similar to consistency between different scenes) that was not observed in either of the patient groups; PCA patients showed a trend towards higher scanpath consistency than controls $(\beta=28.56, t(24)$ $=1.76, p=0.09)$ and tAD patients showed significantly higher consistency than controls $(\beta=$ $32.42, t(25)=2.34, p=0.03$;) (see Figure 5 illustrating between-task similarity for these comparisons). There was no significant difference between PCA and tAD patients $(\beta=3.87$, $t(13)=0.17, p=0.87)$. These results demonstrate that controls modulate their scanpaths in a search task to a greater extent than PCA and tAD patients. Overall, there was a significant interaction $(p=0.006)$ between group and comparison type (non-search/non-search vs search/non-search), reflecting the greater, task-appropriate modulation of controls' scanpaths when viewing scenes under different task demands. 


\subsubsection{Between-observer similarity}

Between-observer similarity measures the similarity of scanpaths made by all the participants of each group when confronted with the same scenes under the same task conditions. Across both the non-search tasks and search tasks (see Figure 5B for means and $\mathrm{Cls}$ ), there was significantly lower scanpath similarity among PCA patients than among either controls $(\beta=20.82, t(24)=3.45, p<0.001$ for search, $\beta=23.53, t(24)=7.88, p<0.001$ for non-search) or tAD patients $(\beta=42.90, t(13)=5.66, p<0.001$ for search and $\beta=18.56$, $t(13)=4.95, p<0.001$ for non-search). Typical AD patients showed a much smaller but still significant difference in scanpath similarity to controls on non-search tasks $(\beta=4.97, t(25)=$ $2.61, p=0.01)$ but showed significantly greater scanpath similarity on the search task $(\beta=$ 22.08, $t(25)=5.43, p<0.001)$. There were also significant overall (all three groups) and pairwise group by condition (search vs nonsearch) interactions (overall $p<0.001, P C A$ vs $t A D$ $p=0.004$, tAD vs healthy controls $p<0.001$ ), and a trend towards a significant interaction in the PCA vs healthy control comparison $(p=0.054)$. These results suggest (i) much greater variability in gaze behaviour among PCA patients, and (ii) a further discrepancy between $P C A$ and $T A D$ performance with tAD scanpath similarity rates differing by a lesser extent on the non-search tasks.

\subsubsection{Low-level salience (normalized scanpath saliency)}

Normalised scanpath saliency was calculated using the low-level salience maps generated for each scene (see Figure 4). PCA patients fixated on more salient regions (mean salience $=1.02, \mathrm{SD}=0.09$ ) than controls (mean $=0.92, \mathrm{SD}=0.08 ; \beta=0.09, t(24)=2.29$, $\mathrm{p}=0.03$ ); tAD patients (mean $=0.97, \mathrm{SD}=0.12$ ) showed an intermediate tendency to fixate high salience regions, not significantly different from that of PCA patients $(\beta=0.03, t(13)=$ $0.43, p=0.67)$ or controls $(\beta=0.06, t(25)=0.93, p=0.36$; see Figure $5 C)$. There was an overall effect of task $(\beta=0.22, t(32)=3.43, p=0.002)$, as regions of lower low-level salience were fixated upon in the search task. However there was no group by task (search vs nonsearch) interaction, suggesting the group differences in salience did not depend on the task condition under which the scene was viewed (see Figure 5C). 
Figure 4. Scanpaths and salience maps. Example scanpaths from two healthy controls (A and $B$ ) and two PCA patients (C and D) demonstrating greater modulation of the scanpath for the search task (green) compared with a non-search task (encode; red) in controls than patients. The between-task similarity is abnormally high for patients ( $C=83 \%$; $D=84 \%)$ relative to controls who view different parts of the scene in a more task-appropriate manner $(A=-17.7 \% ; B=-60.5 \%)$. The highlighted area shows the search target area (all vegetation). Panels $E$ and $F$ show example computer generated low-level salience maps. Low-level salience maps were created using the GBVS toolbox (Harel et al., 2007) with default settings. The tool creates three feature maps for each image (representing variation in colour, intensity and orientation), and then combines these feature maps into a master map representing the computed salience at each pixel. These maps were then normalized to have a mean value of 0 and standard deviation of 1 across pixels. The salience of each fixation was extracted and compared between groups and conditions.

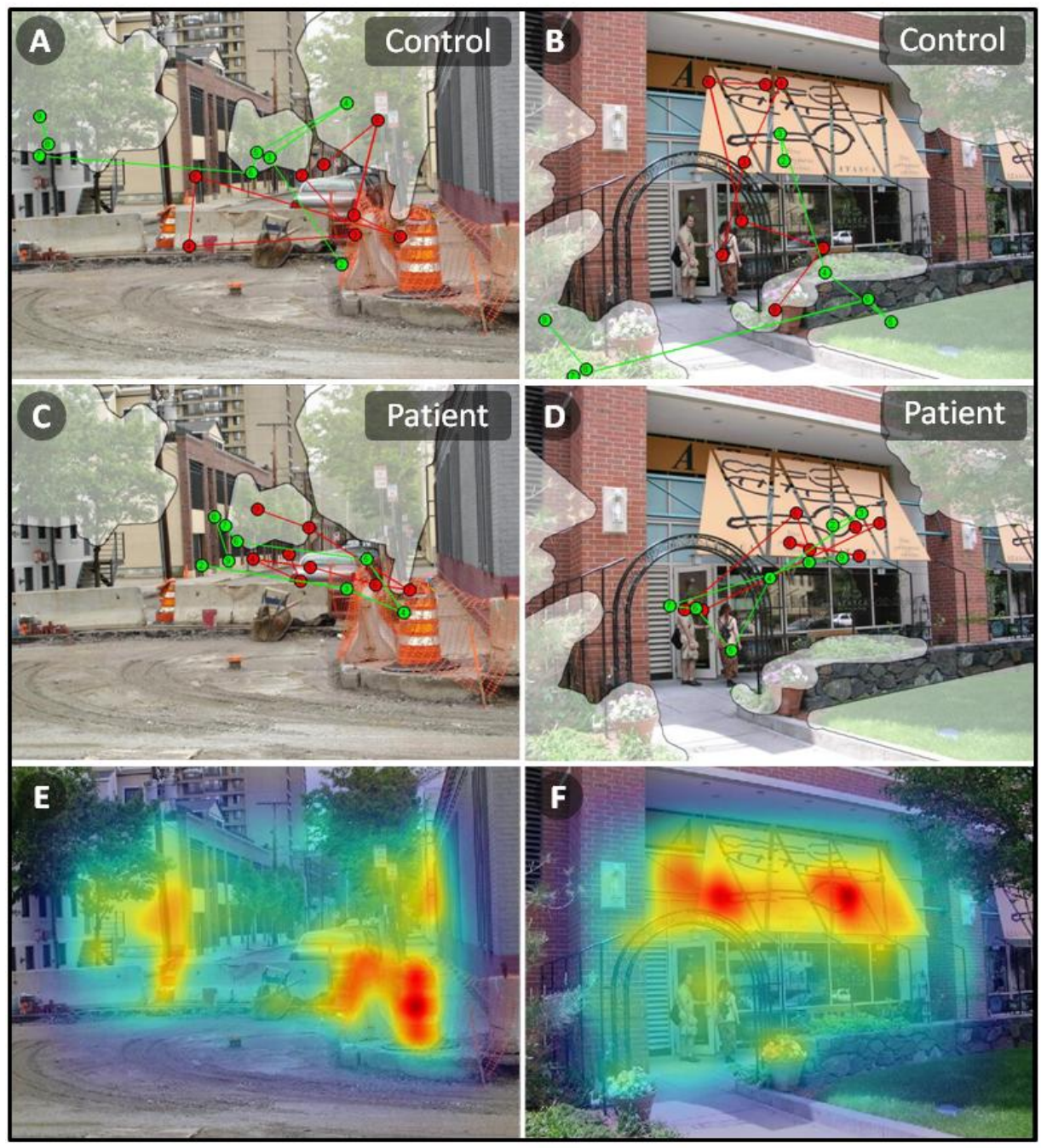


Figure 5. Search task vs non-search task performance in terms of $(A)$ the between task similarity $(B)$ the similarity of scanpaths between participants in the same participant group (between-observer similarity) and (C) scanpath salience. Bars show 95\% confidence intervals.
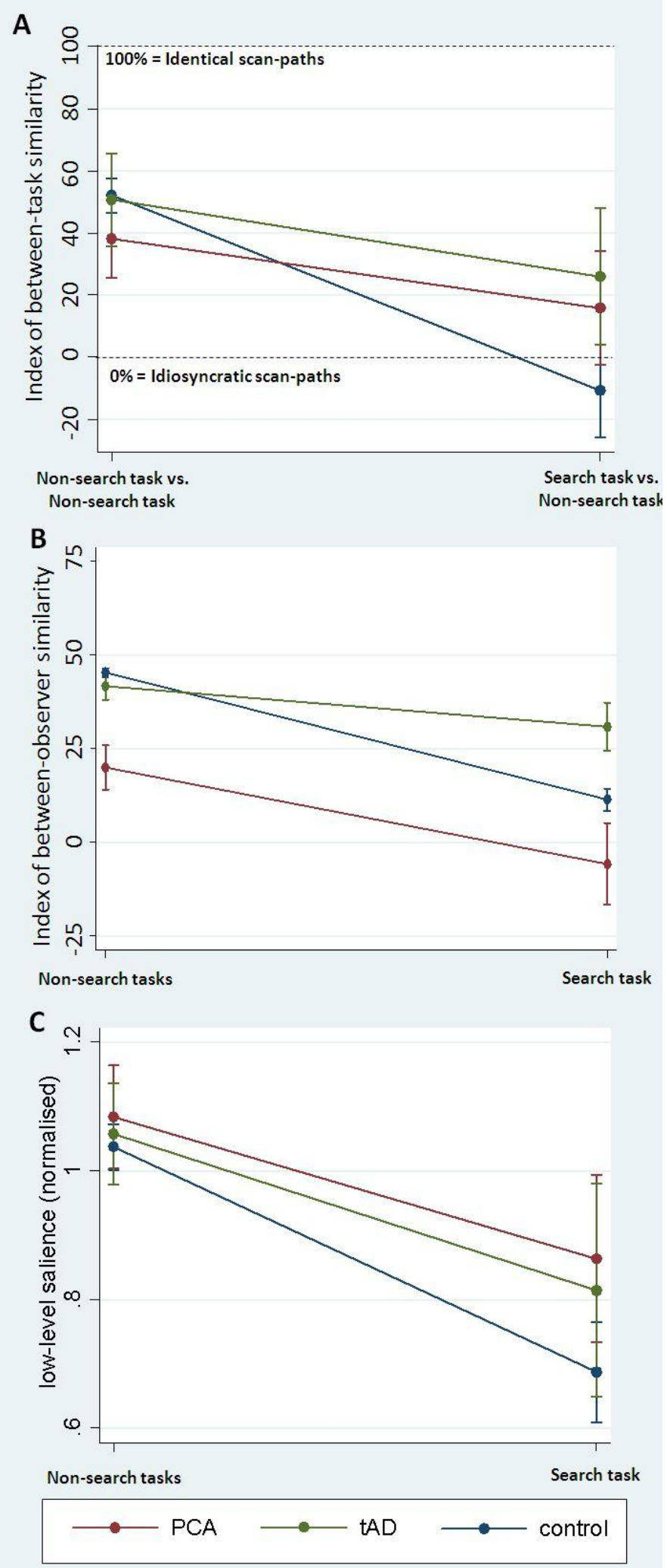


\section{Conclusions}

The current study examined scene perception in individuals with progressive visual impairment associated with posterior cortical atrophy by adapting Yarbus' classic paradigm. Patients' capacity to adapt their eye movement behaviour was tested by viewing scenes under four types of task instruction: encoding, recognition, search and description. Overall PCA patients showed reduced saccade amplitude and gain relative to tAD patients and controls, hence all subsequent analyses were corrected for saccade gain to permit us to explore cognitive influences upon scene perception distinct from discrepancies in basic oculomotor function. Notably tAD patients showed significantly longer fixation durations than controls, but PCA patients showed no such increase. On the search task PCA patients' fixations fell less frequently and more slowly upon targets and there was evidence of an enhanced central fixation bias related to their reduced saccade gain (disappeared when saccadic gain was controlled). PCA patients also exhibited greater difficulty searching for the target 'vegetation' in familiar compared with novel scenes. In the search task there was an overall effect of novelty (when combining the three participant groups), meaning that participants were faster to fixate within the target area, and made more fixations in the target area when search in novel rather than familiar scenes. This may reflect higher motivation for novel scenes than familiar scenes, or a tendency to view previously fixated locations again, even if not relevant to the task at hand. The other three non-search tasks (encoding, recognition and description) contributed primarily to the between-tasks analysis; however it was noted that both PCA and tAD patients showed significantly impaired scene recognition relative to controls which we attribute primarily to poor perception in the PCA group and poor memory in the tAD group given that absence/presence of memory dysfunction formed part of the participant selection criteria.

On the main examination of the effect of test instructions upon scene perception, the results revealed significant differences between the three groups. These differences were most evident when comparing the search task with the other non-search conditions. Across tasks without a search component, PCA patients exhibited lower between-task and betweenobserver similarity ratings than $\mathrm{A} A \mathrm{D}$ patients and controls (i.e. were less consistent in where they were looking), whilst tAD patients showed normal between-task similarity and mildly raised between-observer similarity. By contrast, when comparing search and non-search tasks, it was controls who exhibited the lowest between-task similarity ratings, suggesting they were much better able than PCA or TAD patients to respond appropriately by looking at different target regions of the scene that were relevant to the current task. However, PCA patients continued to show the lowest between-observer similarity ratings suggesting more varied, inconsistent performance among these individuals. These differences were reflected in significant group by task interaction effects. PCA patients also had a significant tendency to fixate upon more salient parts of the scenes than controls irrespective of the viewing task, whilst tAD patients showed a milder tendency intermediate between that of PCA patients and controls.

The two research objectives of this study of naturalistic scene perception in posterior cortical atrophy and typical AD were complementary. The experimental manipulations were designed to help us to better understand how PCA and TAD patients perceive the real world, and to explain some of the characteristic features and inconsistencies of their everyday perception. On the other hand, understanding task-related effects upon these patients' viewing behaviour sheds light on the mechanisms by which high level cognitive schemes interact with low level perception. These two complementary goals are considered in more detail below.

\subsection{Real-world scene perception in PCA}

Despite visual dysfunction being the primary feature of the PCA syndrome, there is very little understanding of how individuals with the condition actually perceive the world. The current study provides evidence that PCA scene perception is characterized (in contrast to TAD and 
healthy controls) by saliency-dependence, a reduced ability to fixate task-relevant areas and reduced consistency between observers. In particular, two features of the observed performance may help explain why patients and carers frequently report variability and inconsistency in everyday vision (e.g. completely failing to perceive the saltcellar on the table in front of them when asked to pass the salt, and then picking up and using it appropriately soon after). First, between-task consistency of fixations and scan paths was abnormally high, reflecting inability to adjust flexibly to environmental contingencies. Some goal-oriented or externally-directed conditions (e.g. actively searching for a particular object) may place much heavier demands on this capacity than other more passive or self-directed conditions (e.g. observing the object during free-viewing). Second, between-observer consistency was abnormally low; this may be because inconsistencies in the basic visual processing of item features leads to the formation and retrieval of distorted or irrelevant higher level scene schema.

A detailed functional account of PCA patients' real-world perception as it relates to everyday tasks is critical for improving clinical management. One clinically-relevant extension of the current study would be to progress from scene pictures to more naturalistic settings in which the influence of not only external test instructions but the behavioural goals of the observer can be examined. Analyses of eye movements during naturalistic actions (e.g. making a sandwich, driving, playing cricket) have revealed the crucial role of prior knowledge (see Hayhoe \& Ballard, 2005 for a review). Whilst we have assumed that scene schema knowledge for the simple tasks and static scene photographs in the current study (e.g. knowing grass is more likely to be found on the ground than on walls) is relatively preserved, it remains to be seen whether relevant prior knowledge is available or can be successfully transformed into computations for the planning and guidance of eye movements in more dynamic situations (e.g. anticipating the bounce point of a ball, or the moment of contact when reaching out to grasp a door handle). Understanding patients' perception of naturalistic settings rather than scene depictions will also involve consideration of a wider range of eye movements and control circuits (e.g. the predictive component of the pursuit system; Barnes \& Collins, 2008; Tabata et al., 2008).

\subsection{High- and low-level influences on scene perception}

Scene perception is a complex process in which eye movements are influenced by knowledge structures (top-down control), image features (bottom-up processes) and the interaction between these mechanisms. The experimental paradigm used in the current study was designed to manipulate knowledge-driven control whilst holding image features constant. The pattern of results obtained (as outlined above) suggest that PCA patients exhibit a loss of the capacity to adapt their eye movement behaviour to current task demands. The fact that this loss of task-appropriate modulation was most evident when contrasting search with non-search tasks may indicate that PCA patients have greater difficulty engaging particular more active modes of real-world scene perception relative to more passive viewing conditions. The loss of the capacity to adapt eye movement behaviour to current task demands is unlikely to be explained by basic oculomotor dysfunction (because saccade gain was controlled for, and all between-task comparisons employed a within-subjects design), failure to understand the test instructions (which were simplified from the original Yarbus paradigm), stimulus properties (as the identical scenes were viewed under each task condition), or a non-specific 'dementia' effect (as PCA performance diverged from that of typical AD patients in a number of respects).

Although the primary experimental manipulation was of task knowledge (i.e. test instructions), other forms of knowledge are known to influence eye movement control. These include scene schema knowledge (e.g. vegetation is usually at the edge rather than centre of carriageways) and short-term episodic scene knowledge (e.g. the tree was in the top left corner; see Henderson \& Ferreira, 2004). PCA patients' well-preserved semantic knowledge of the world means there is no reason to suspect deficits in scene schema knowledge. 
However, short-term episodic scene knowledge may well have been compromised by a combination of factors including visuoperceptual and visuospatial deficits (see background neuropsychology in the supplementary material (S2)) and additional impairments in visual short term memory (VSTM; Hollingworth and Henderson, 2002; Hollingworth et al., 2001; Tatler et al., 2003; Todd and Marois, 2004) leaving an unreliable or incomplete trace of feature identity and location within the scene. Such an impairment of short-term episodic scene knowledge or VSTM could account for several features of the PCA patients' performance, including the significant increase in time taken for PCA patients to fixate within a target area for the first time during the search task. As the search task was the third task administered, participants had two prior opportunities (during encoding and recognition) to view the (familiar) scenes and to construct a short-term episodic trace of the scene which, for controls, could support the rapid re-fixation of task-relevant target areas. This argument is strengthened by the particular disparity of the proportion of fixations in target areas between PCA patients and both controls and tAD patients when searching familiar scenes (for which a short-term episodic trace would have been established) relative to novel scenes (for which no such trace was available).

A related but distinct explanation of $T A D$ patient performance on these tasks may also be offered. Whilst we speculate that PCA patients exhibit an impairment of short-term episodic scene knowledge owing to poor perceptual encoding, we suggest that the milder disruption of scanpath modulation in tAD may reflect impairment of short-term episodic scene knowledge owing to poor mnemonic encoding and/or retrieval of (relatively accurately) perceived scene information. This interpretation is analogous to the suggestion that PCA and $\mathrm{TAD}$ patients tend to achieve equivalently poor performance on some episodic memory tasks for different reasons: poor perception/registration of stimuli (PCA) versus poor encoding and retrieval (tAD). Future studies of PCA and TAD scene perception may explore these distinct but related explanations by comparison of immediate and delayed scene recognition, with the prediction that TAD but not PCA patients should exhibit loss of shortterm episodic scene knowledge over the delay resulting in further reductions in within-task and within-observer scanpath similarity measures.

A better understanding of the interactions between knowledge-driven and image feature control of eye movements remains a critical area in eye movement research. Although the current experiment was designed to hold image features constant by means of the comparison of task performance across identical scenes, a computer-based image saliency map was used to probe for any differences in saliency-biases across different viewing tasks. However, no statistical interaction was found between the salience of image features and the task demands (PCA patients showed a greater tendency to focus on high salience regions irrespective of task c.f. Foulsham et al., $2009 \& 2011$ ). Whilst this result may simply reflect the primacy of basic visual function in determining PCA patients' scene perception, the current paradigm could be adapted to involve two search tasks in which the salience or other visual properties of the targets are varied.

Finally, we considered some limitations of the study. First, it is worth noting that this experiment tested only one aspect of visual search, namely searching for vegetation within scenes. This is a relatively narrow perspective on search tasks and may limit the generalizability of the results. Future experiments should investigate search for different features such as particular objects, people or areas defined by a basic feature such as colour or luminance, and perhaps investigate search for multiple rather than single targets. Such tasks may help to further elucidate the nature of everyday perception in PCA and other individuals with dementia-related visual impairment. Second, interpretation of scan path modulation is perhaps a topic for debate. For example in the comparison of recognition and description tasks, PCA patients showed lower consistency (and therefore greater modulation) than controls and tAD patients. We would suggest that the lower consistency in patients than controls in this case may represent a disorganised approach. In the description 
condition this may be driven by a search for items that they are able to describe in spite of their word finding difficulties, rather than deliberate modulation to achieve a required goal.

In conclusion, this study provides a detailed characterization of scene perception abilities in posterior cortical atrophy through investigation of gaze patterns. A better appreciation of gaze control mechanisms in natural vision will form a critical basis for attempts to develop strategies and aids to reduce the widespread impact of dementia-related visual dysfunction upon activities of daily living and quality of life not just in PCA but in typical Alzheimer's disease and Dementia with Lewy Bodies more generally.

\section{Acknowledgements}

We would like to thank all the participants for their incredibly valuable contribution. Thanks also to Professor Graham Barnes for his comments on an earlier draft of this manuscript, and to Dr Jonathon Schott for his advice on biomarker interpretation.

\section{Funding}

This work was undertaken at UCLH/UCL which received a proportion of funding from the Department of Health's NIHR Biomedical Research Centres funding scheme. The Dementia Research Centre is an Alzheimer's Research UK Co-ordinating Centre (Grant no. ARUKSRF2013-8). The Dementia Research Centre is supported by Alzheimer's Research UK, Brain Research Trust (Grant no. EQW 121 301), and The Wolfson Foundation. SC is supported by an ARUK Senior Research Fellowship and an Economic and Social Research Council (ESRC)/National Institute for Health Research (NIHR) grant (ES/K006711/1). TS is supported by an ARUK Research Fellowship. The eye tracking equipment was funded by an ARUK equipment grant. This work was supported by the NIHR Queen Square Dementia Biomedical Research Unit. The views expressed are those of the authors and not necessarily those of the NHS, the NIHR or the Department of Health. No funding source had involvement in study design, data collection, analysis or interpretation of data, writing of the report or the decision to submit the article for publication.

\section{References}

Ballard, D. H., Hayhoe, M. M., \& Pelz, J. B. (1995). Memory representations in natural tasks. Journal of Cognitive Neuroscience, 7(1), 66-80.

Barnes, G. R., \& Collins, C. J. S. (2008). Internally generated smooth eye movement: its dynamic characteristics and role in randomised and predictable pursuit. Progress in Brain Research, 171(08), 441-9.

Benson, D. F., Davis, R. J., \& Snyder, B. D. (1988). Posterior cortical atrophy. Archives of Neurology, 45(7), 789-93.

Buswell, G. T. (1935). How People Look at Pictures: A Study of the Psychology of Perception in Art. Chicago: University of Chigago Press.

Castelhano, M. S., Mack, M. L., \& Henderson, J. M. (2009). Viewing task in fluences eye movement control during active scene perception, 9, 1-15.

Charles, R. F., \& Hillis, A. E. (2005). Posterior cortical atrophy: Clinical presentation and cognitive deficits compared to Alzheimer's disease. Behavioural Neurology, 16, 15-23.

Chen, X., \& Zelinsky, G. J. (2006). Real-world visual search is dominated by top-down guidance. Vision Research, 46(24), 4118-33.

Crutch, S. J., Lehmann, M., Schott, J. M., Rabinovici, G. D., Rossor, M. N., \& Fox, N. C. (2012). Posterior cortical atrophy. Lancet Neurology, 11(2), 170-8.

DeAngelus, M., \& Pelz, J. B. (2009). Top-down control of eye movements: Yarbus revisited. Visual Cognition, 17(6-7), 790-811.

Dubois, B., Feldman, H. H., Jacova, C., Cummings, J. L., Dekosky, S. T., Barberger-Gateau, P., ... Scheltens, P. (2010). Revising the definition of Alzheimer's disease: a new lexicon. Lancet Neurology, 9(11), 1118-27. 
Dubois, B., Feldman, H. H., Jacova, C., Dekosky, S. T., Barberger-Gateau, P., Cummings, J., ... Scheltens, P. (2007). Research criteria for the diagnosis of Alzheimer's disease: revising the NINCDS-ADRDA criteria. Lancet Neurology, 6(8), 734-46.

Ehinger, K., Hidalgo-Sotelo, B., Torralba, A., \& Oliva, A. (2009). Modeling Search for People in 900 Scenes: A combined source model of eye guidance. Visual Cognition, 17(6-7), 945-978.

Foulsham, T., Barton, J. J. S., Kingstone, A., Dewhurst, R., \& Underwood, G. (2009). Fixation and saliency during search of natural scenes: the case of visual agnosia. Neuropsychologia, 47(8-9), 1994-2003.

Foulsham, T., Barton, J. J. S., Kingstone, A., Dewhurst, R., \& Underwood, G. (2011). Modeling eye movements in visual agnosia with a saliency map approach: bottom-up guidance or top-down strategy? Neural Networks, 24(6), 665-77.

Foulsham, T., Dewhurst, R., Nyström, M., Johansson, R., \& Underwood, G. (2012). Comparing scanpaths during scene encoding and recognition $\square$ : A multi-dimensional approach.

Harel, J., Koch, C., \& Perona, P. (2007). Graph-Based Visual Saliency. Advances in Neural Information Processing Systems (NIPS), 19, 545-552.

Hayhoe, M. M., \& Ballard, D. (2005). Eye movements in natural behavior. Trends in Cognitive Sciences, 9(4), 188-94.

Henderson, J. M. (2007). Regarding Scenes. Current Directions in Psychological Science, $16(4), 219-222$.

Henderson, J. M. (2011). Eye movements and scene perception. In I. D. Gilchrist, S. P. Liversedge, \& S. Everling (Eds.), The Oxford handbook of eye movements (pp. 593606). Oxford, UK: Oxford University Press.

Henderson, J. M., \& Ferreira, F. (2004). Scene perception for psycholinguists. In J. M. Henderson \& F. Ferreira (Eds.), The interface of language, vision, and action: Eye movements and the visual world (pp. 1-58). New York: Psychology Press.

Henderson, J. M., Malcolm, G. L., \& Schandl, C. (2009). Searching in the dark: cognitive relevance drives attention in real-world scenes. Psychonomic Bulletin \& Review, 16(5), 850-6.

Henderson, J. M., Nuthmann, A., \& Luke, S. G. (2013). Eye movement control during scene viewing: immediate effects of scene luminance on fixation durations. Journal of Experimental Psychology. Human Perception and Performance, 39(2), 318-22.

Hollingworth A, Henderson JM. Accurate visual memory for previously attended objects in natural scenes. J. Exp. Psychol. Hum. Percept. Perform. 2002; 28: 113-136.

Hollingworth A, Williams CC, Henderson JM. To see and remember: Visually specific information is retained in memory from previously attended objects in natural scenes. Psychon. Bull. Rev. 2001; 8: 761-768.

Itti, L., \& Koch, C. (2000). A saliency-based search mechanism for overt and covert shifts of visual attention. Vision Research, 40(10-12), 1489-506.

Itti, L., \& Koch, C. (2001). Computational modelling of visual attention. Nature Reviews. Neuroscience, 2(3), 194-203.

Itti, L., Koch, C., \& Niebur, E. (1998). A model of saliency-based visual attention for rapid scene analysis. IEEE Transactions on Pattern Analysis and Machine Intelligence, 20(11), 1254-1259.

Koch, C., \& Ullman, S. (1985). Shifts in selective visual attention: towards the underlying neural circuitry. Human Neurobiology, 4(4), 219-27.

Lehmann, M., Crutch, S. J., Ridgway, G. R., Ridha, B. H., Barnes, J., Warrington, E. K., ... Fox, N. C. (2011). Cortical thickness and voxel-based morphometry in posterior cortical atrophy and typical Alzheimer's disease. Neurobiology of Aging, 32(8), 1466-76.

Machner, B., Dorr, M., Sprenger, A., von der Gablentz, J., Heide, W., Barth, E., \& Helmchen, C. (2012). Impact of dynamic bottom-up features and top-down control on the visual exploration of moving real-world scenes in hemispatial neglect. Neuropsychologia, 50(10), 2415-25. 
Mannan, S. K., Kennard, C., \& Husain, M. (2009). The role of visual salience in directing eye movements in visual object agnosia. Current Biology, 19(6), R247-8.

McMonagle, P., Deering, F., Berliner, Y., \& Kertesz, A. (2006). The cognitive profile of posterior cortical atrophy. Neurology, 66(3), 331-8.

Mendez, M., Ghajarania, M., \& Perryman, K. M. (2002). Posterior cortical atrophy: clinical characteristics and differences compared to Alzheimer's disease. Dementia and Geriatric Cognitive Disorders, 14(1), 33-40.

Navalpakkam, V., \& Itti, L. (2005). Modeling the influence of task on attention. Vision Research, 45(2), 205-31.

Parkhurst, D., Law, K., \& Niebur, E. (2002). Modeling the role of salience in the allocation of overt visual attention. Vision Research, 42(1), 107-23.

Rao, R. P. N., Zelinsky, G. J., Hayhoe, M. M., \& Ballard, D. H. (2002). Eye movements in iconic visual search. Vision Research, 42(11), 1447-63.

Renner, J. A., Burns, J. M., Hou, C. E., McKeel, D. W., Storandt, M., \& Morris, J. C. (2004). Progressive posterior cortical dysfunction: a clinicopathologic series. Neurology, 63(7), 1175-80.

Tabata, H., Miura, K., \& Kawano, K. (2008). Trial-by-Trial Updating of the Gain in Preparation for Smooth Pursuit Eye Movement Based on Past Experience in Humans, 747-758.

Tang-Wai, D. F., Graff-Radford, N. R., Boeve, B. F., Dickson, D. W., Parisi, J. E., Crook, R., ... Petersen, R. C. (2004). Clinical, genetic, and neuropathologic characteristics of posterior cortical atrophy. Neurology, 63(7), 1168-74.

Tatler, B. W. (2007). The central fixation bias in scene viewing $\square$ : Selecting an optimal viewing position independently of motor biases and image feature distributions, 7, 1-17. doi:10.1167/7.14.4.Introduction

Tatler, B. W., Gilchrist, I. D., \& Land, M. F. (2005). Visual memory for objects in natural scenes: from fixations to object files. The Quarterly Journal of Experimental Psychology. A, Human Experimental Psychology, 58(5), 931-60.

Tatler BW, Gilchrist ID, Rusted J. The time course of abstract visual representation. Perception 2003; 32: 579-592.

Tatler, B. W., Wade, N. J., Kwan, H., Findlay, J. M., \& Velichkovsky, B. M. (2010). Yarbus, eye movements, and vision. I-Perception, 1(1), 7-27.

Todd JJ, Marois R. Capacity limit of visual short-term memory in human posterior parietal cortex. Nature 2004; 428: 751-4.

Torralba, A., Oliva, A., Castelhano, M. S., \& Henderson, J. M. (2006). Contextual guidance of eye movements and attention in real-world scenes: the role of global features in object search. Psychological Review, 113(4), 766-86.

Warrington, E. K. (1996). The Camden Memory Tests. Hove: Psychology Press.

Yarbus, A. L. (1967). Eye Movements and Vision. New York: Plenum Press. 


\section{Supplementary Material}

S1. Scene stimuli

These 30 photographic images of street scenes were selected from a readily available previously studied dataset (Ehinger et al., 2009).
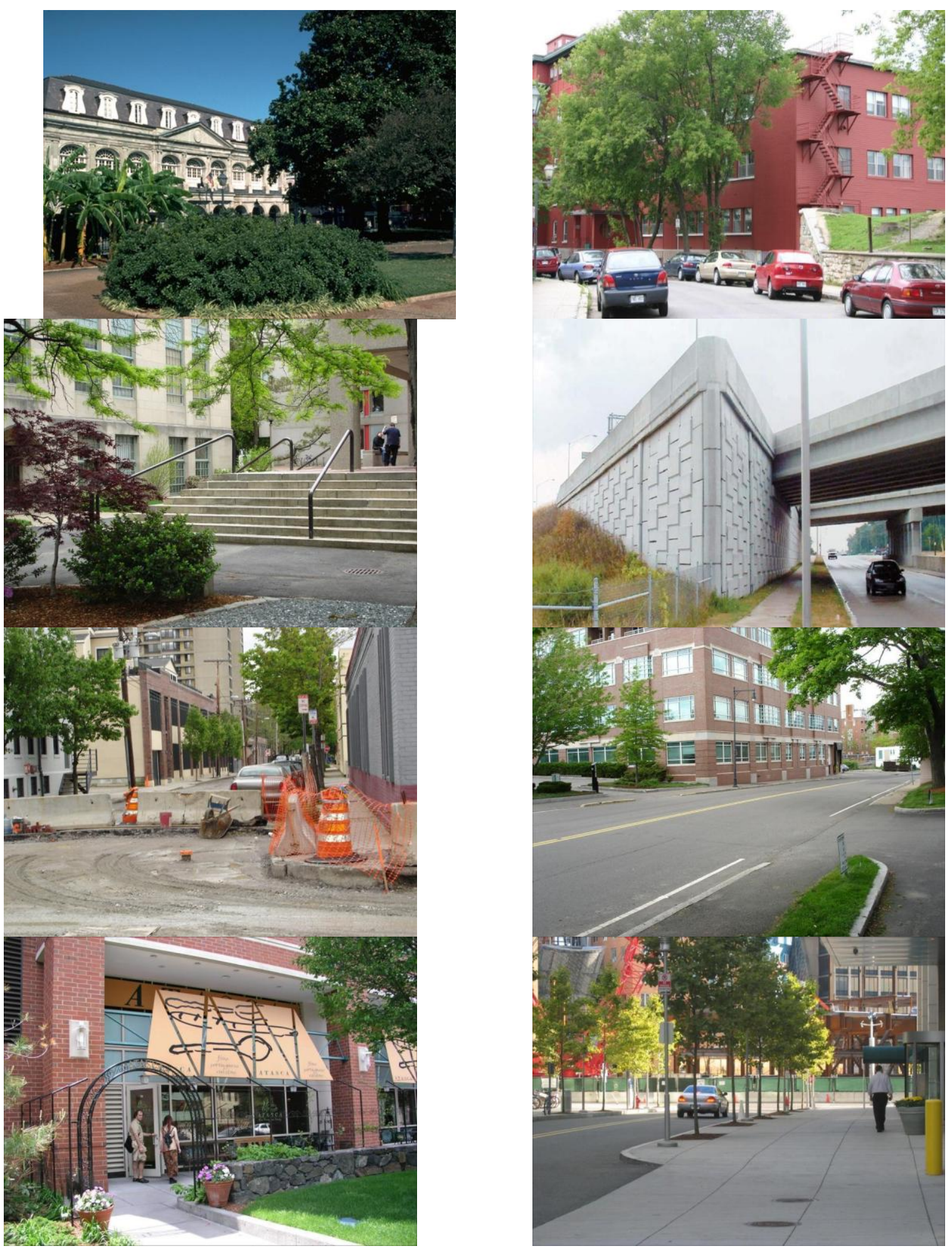

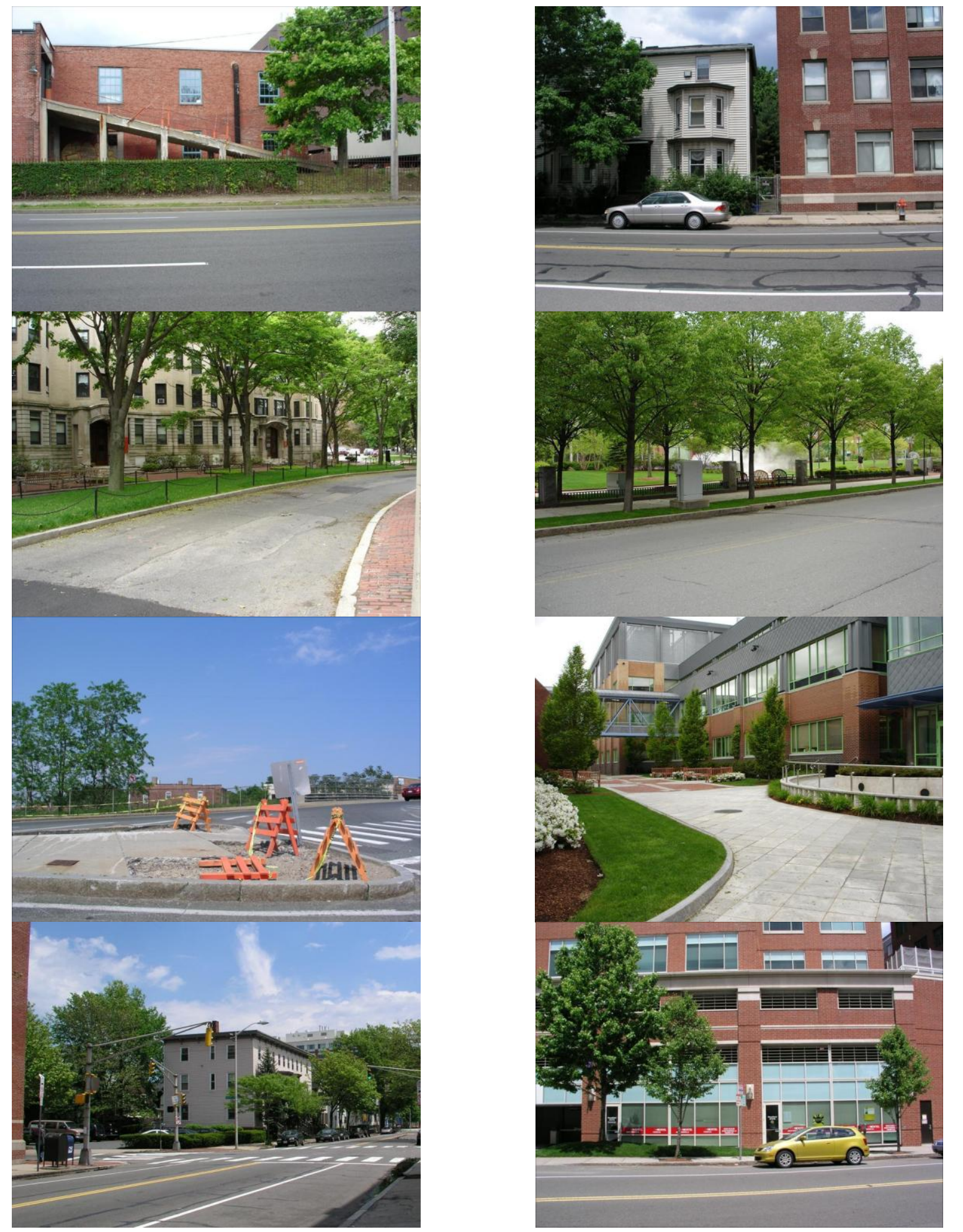

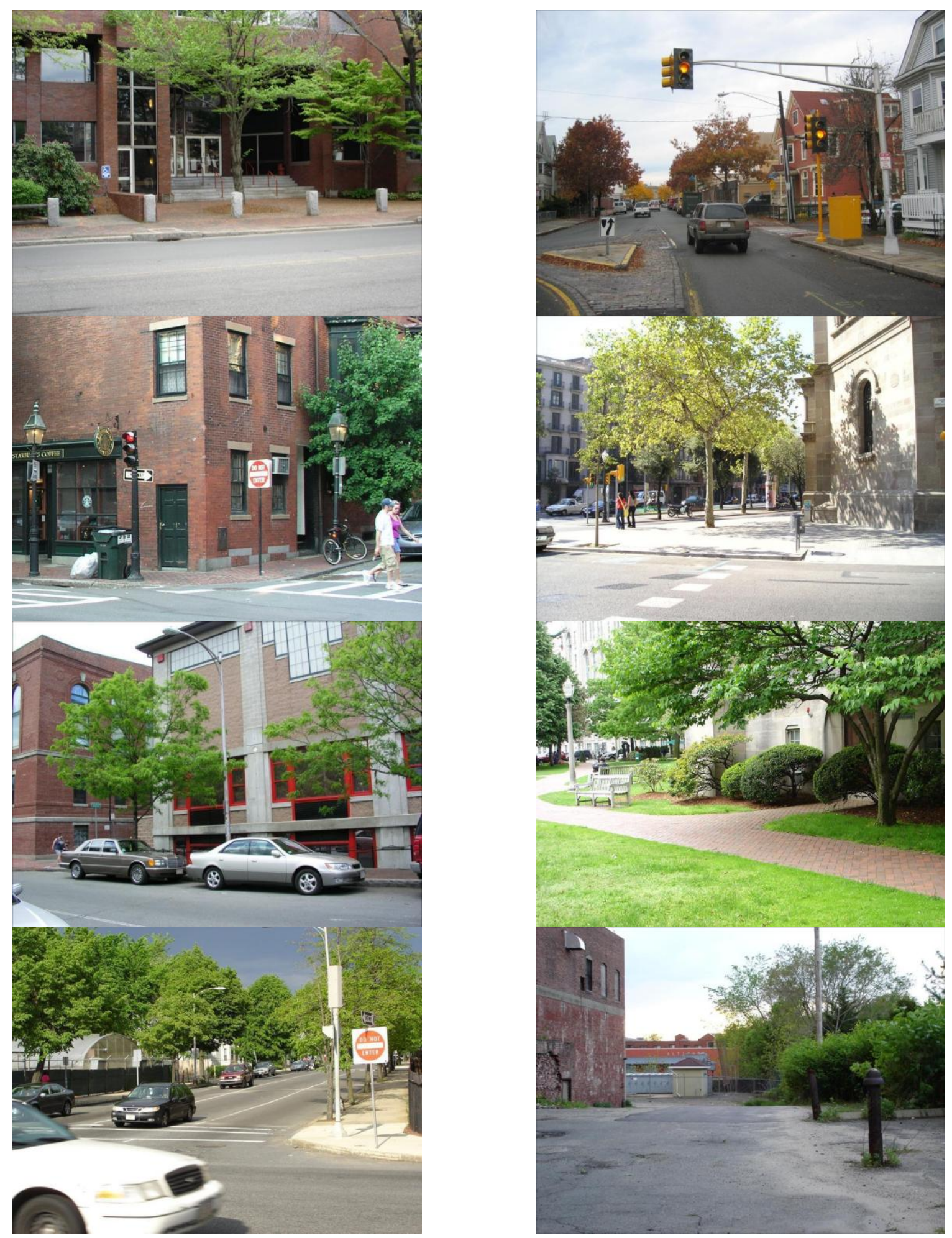


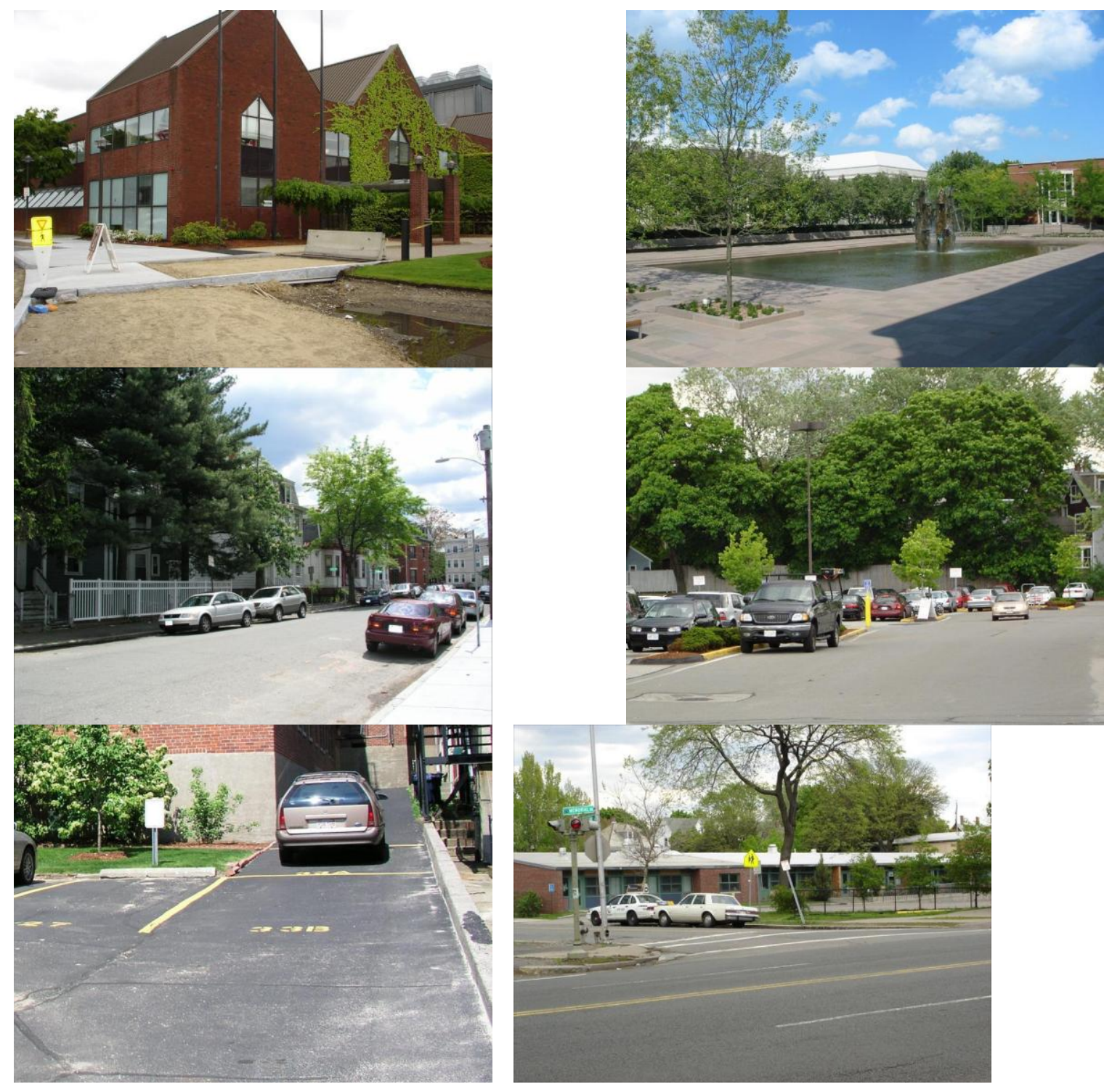


S2. Background psychology

\begin{tabular}{|c|c|c|c|c|c|c|c|c|c|c|}
\hline Patient number: & 1 & 2 & 3 & 4 & 5 & 6 & 7 & $\begin{array}{l}\text { PCA } \\
\text { Mean } \\
\text { (SD) }\end{array}$ & $\begin{array}{l}N \text { below } \\
5 \text { th \%ile }\end{array}$ & $\begin{array}{l}\text { Normative } \\
\text { Mean (SD) }\end{array}$ \\
\hline Age & 63.2 & 58.6 & 51.8 & 70.4 & 53.8 & 56.4 & 57.9 & $58.9(6.3)$ & - & - \\
\hline Gender & $M$ & $\mathrm{~F}$ & $\mathrm{~F}$ & $\mathrm{~F}$ & $\mathrm{~F}$ & $\mathrm{~F}$ & $M$ & $5 f, 2 m$ & - & - \\
\hline Disease duration (years) & 7 & 3 & 5 & 2 & 2 & 2 & 2 & $3.4(1.9)$ & - & - \\
\hline $\operatorname{MMSE}(/ 30)^{\mathrm{a}}$ & 23 & 24 & 22 & 26 & 18 & 24 & 21 & $22.6(2.6)$ & - & - \\
\hline sRMT words $(/ 25)^{b}$ & 20 & 25 & 24 & 25 & 21 & 21 & 20 & $22.3(2.3)$ & 0 & $23.7(1.8)$ \\
\hline sRMT faces $\quad(/ 25)^{b}$ & 23 & 15 & 19 & 22 & 25 & 22 & 22 & $21.1(3.2)$ & 2 & $22.8(1.9)$ \\
\hline $\begin{array}{l}\text { Naming from description } \\
(/ 20)^{c}\end{array}$ & 10 & 18 & 15 & 19 & 4 & NT & 17 & $13.8(5.8)$ & 3 & $18.9(1.5)$ \\
\hline Calculation $(/ 26)^{d}$ & 19 & 9 & 9 & 17 & 9 & 16 & 9 & $12.6(4.5)$ & 4 & $20.7(3.1)$ \\
\hline Spelling $(/ 30)^{\mathrm{e}}$ & 7 & 16 & 7 & 18 & 5 & 8 & 5 & $9.4(5.3)$ & 5 & $19.5(6.5)$ \\
\hline Object decision $(/ 20)^{f}$ & 14 & 9 & 12 & 16 & 16 & 14 & 7 & $12.6(3.5)$ & 5 & $17.7(1.9)$ \\
\hline Number location $(/ 10)^{\dagger}$ & 7 & 0 & 0 & 2 & 6 & 3 & 0 & $2.6(2.9)$ & 7 & $9.4(1.1)$ \\
\hline Concrete Synonyms $(/ 30)^{\mathrm{g}}$ & 16 & 23 & 21 & 24 & 23 & NT & 23 & $21.7(2.9)$ & 0 & $20.8(3.0)$ \\
\hline
\end{tabular}

Raw scores for each patient are presented, with mean and standard deviation scores for the PCA patient group and relevant normative data. NT $=$ not tested.

Normative data samples: a mini-mental state examination( Folstein et al. 1975); b Short Recognition Memory Test (Warrington 1996); c Randlesome (unpublished data $N=100$ ); $d$ Crutch (unpublished data); e Baxter spelling test (Baxter and Warrington 1994); $f$ Visual Object and Space Perception battery (Warrington and James 1991); g Synonyms test (Warrington et al., 1998). 
S3. Supplementary references

Baxter, D. M., \& Warrington, E. K. (1994). Measuring dysgraphia: a graded-difficulty spelling test. Behavioural Neurology, 7, 107-116.

Folstein, M. F., Folstein, S. E., \& McHugh, P. R. (1975). "Mini-mental state". A practical method for grading the cognitive state of patients for the clinician. Journal of Psychiatric Research, 12(3), 189-98.

Warrington, E. K. (1996). The Camden Memory Tests. Hove: Psychology Press.

Warrington, E. K., \& James-Galton, M. (1991). The Visual Object and Space Perception Battery. Bury St. Edmunds: Thames Valley Test Company.

Warrington, E. K., McKenna, P., \& Orpwood, L. (1998). Single Word Comprehension: A Concrete and Abstract Word Synonym Test. Neuropsychological Rehabilitation, 8(2), 143-154. 